(Abb. 1 C). An Stelle des Dubletts erscheint ein neues Singlett mit einer Linienbreite von 17 Gauss und einem Aufspaltungsfaktor $g=2,0017 \pm 0,0002$ (Abb. 1 C). Der Übergang des Dubletts in das neue Singlett findet um so leichter statt, je höher die Alkalikonzentration ist.

Auch eingefrorene Lösungen von schwerem Wasser zeigen in ihren ESR-Spektren ein ähnliches Verhalten. Abb. $1 \mathrm{D}$ zeigt das Triplett des OD-Radikals in bestrahltem, neutralem, schwerem Eis bei $77^{\circ} \mathrm{K}^{10 a, b, d}$. Wird alkalisches, schweres Eis bestrahlt (Abb. $1 \mathrm{E}$ ), so erscheint auch hier neben dem Triplett des OD-Radikals das Singlett des solvatisierten Elektrons (vgl. Abb. 1 B). Neben dem Triplett in Abb. $1 \mathrm{E}$ ist außerdem das neue Singlett bei höherer Magnetfeldstärke zu beobachten. Durch wiederholtes, kurzzeitiges Tempern auf 100 bis $110^{\circ} \mathrm{K}$ verschwindet das Triplett des OD-Radikals weitgehend, während das neue Singlett deutlich in den Vordergrund tritt (Abb. 1 F). Auch hier wird das Singlett des solvatisierten Elektrons durch das Erwärmen nicht wesentlich beeinflußt.

Durch das Tempern der Proben tritt eine Verände. rung der ESR-Signale des OH- bzw. OD-Radikals ein. Die resultierenden Spektren enthalten nur noch zwei Singletts, unabhängig davon, ob schweres oder leichtes Eis verwendet wird (vgl. Abb. $1 \mathrm{C}$ und F).

Das neue Singlett kann wegen seiner geringen Halbwertsbreite nicht durch Austauschverbreiterung aus dem Dublett bzw. dem Triplett entstanden sein. Auch eine Austauschverengung durch Ladungsaustausch nach (6a) und $(6 \mathrm{~b})$, oder durch Protonenresonanz nach (7) ist als Ursache für das Auftreten des neuen Singlett-Signals unwahrscheinlich, weil es gegenüber dem Dublett des $\mathrm{OH}$-Radikals eine verschobene Lage hat.

$$
\begin{aligned}
\mathrm{HO}^{\bullet}+{ }^{\ominus} \mathrm{OH} \rightleftharpoons & \mathrm{HO}^{\ominus}+{ }^{\ominus} \mathrm{OH}, \\
\mathrm{HO}^{\circ} \ldots \mathrm{HO}^{\ominus} \rightleftharpoons & \mathrm{HO}^{\ominus} \ldots \mathrm{HO}^{\circ}, \\
{ }^{\circ} \mathrm{OH} \ldots{ }^{\ominus} \mathrm{OH} \rightleftharpoons & \mathrm{O}^{\ominus} \ldots \mathrm{H}-\mathrm{OH} .
\end{aligned}
$$

Die Deutung der Befunde geht davon aus, daß das neue Singlett durch Tempern der Proben auf 100 bis $110^{\circ} \mathrm{K}$ entsteht. Bei diesen Temperaturen werden $\mathrm{OH}$ - und OD-Radikale beweglich ${ }^{9}$. Wir nehmen an, daß die beim Erwärmen des bestrahlten, alkalischen Eises beweglich gewordenen $\mathrm{OH}-$ bzw. OD-Radikale mit den $\mathrm{OH}^{\ominus}$. Ionen bzw. $\mathrm{OD}^{\ominus}$-Ionen nach $(8 \mathrm{a})$ und $(8 \mathrm{~b})$ reagieren können und die basische Form des OH- bzw. OD-Radikals bilden:

$$
\begin{gathered}
\mathrm{HO}^{\ominus}+\mathrm{HO}^{\ominus} \rightarrow \mathrm{O}^{\ominus}+\mathrm{H}_{2} \mathrm{O}, \\
\mathrm{DO}^{\bullet}+\mathrm{DO}^{\ominus} \rightarrow \mathrm{O}^{\ominus}+\mathrm{D}_{2} \mathrm{O} .
\end{gathered}
$$

Das Auftreten des neuen Singletts in den ESR-Spektren von bestrahltem, alkalischem, leichtem und schwerem Eis ist nach dieser Vorstellung mit dem Auftreten des $\mathrm{O}^{\ominus}$-Radikalions verknüpft. Nach HART, Gordon und Hutchinson ${ }^{4}$ beträgt der $p_{\mathrm{K}}$-Wert des $\mathrm{O}^{\ominus}$-Radikalions 11,8. In Übereinstimmung damit tritt das $\operatorname{dem} \mathbf{O}^{\ominus}$. Radikalion zugeordnete Singlett nur dann auf, wenn die Alkalikonzentration im Eis größer als $10^{-2} \mathrm{Mol} / 1$ ist.

Die ESR-Spektren haben wir im Institut für Strahlenbiologie des Kernforschungszentrums Karlsruhe aufgenommen. Wir danken Herrn Prof. Dr. K. G. Zimmer und Herrn Dr. A. Müller für ihre freundliche Hilfe.

\section{Unsymmetrie in der Intensitätsverteilung charakteristischer Energieverluste}

\section{Von M. Creuzburg}

Institut für Angewandte Physik der Universität Hamburg (Z. Naturforschg. 18 a, 101-102 [1963]; eingeg. am 20. Dezember 1962)

An Silicium und Germanium werden bei Durchstrahlung mit mittelschnellen Elektronen $(50 \mathrm{keV})$ charakteristische Energieverluste von ca. $17 \mathrm{eV}$ bzw. ca. $16 \mathrm{eV}$ gefunden, die man als Volumenplasmaverluste deutet. Erniedrigt man die Dicke der durchstrahlten Schicht, so verlieren diese Verluste an Intensität. Bei kleineren Schichtdicken als ca. $100-150 \AA$ erkennt man im Energieverlustspektrum kein Maximum bei $17 \mathrm{eV}$ bzw. bei $16 \mathrm{eV}$ mehr. Statt dessen tritt bei ca. $5 \mathrm{eV}$ ein neuer relativ scharfer Energieverlust hervor. Deutet man diesen Verlust als Oberflächenverlust, so ist die Beobachtung verständlich, daß er bei größerer Schichtdicke von den dann intensiveren Volumenplasmaverlusten überdeckt wird. Ein ähnliches Verhalten gegenüber Verände- rung der Schichtdicke ließ den 6 eV-Aluminium-Verlust und den 3,6 eV-Silber-Verlust als Oberflächenplasmaverluste erkennen ${ }^{1}$.

Eine weitere Eigenschaft von Oberflächenplasmaverlusten stellt nach STERN und FerReLl ${ }^{2}$ die Winkelabhängigkeit ihres differentiellen Wirkungsquerschnittes dar, der durch

$$
\sigma(\vartheta)=A \cdot\left[\vartheta /\left(B^{2}+\vartheta^{2}\right)^{2}\right]
$$

gegeben ist. Es wurde daher die Intensität des $5 \mathrm{eV}$ Verlustes in Abhängigkeit vom Streuwinkel $\vartheta$ gemessen. Das Meßergebnis ist wegen des raschen Abfalles in dieser Verteilung durch das endliche Winkelauflösungsvermögen der Apparatur (Halbwertsbreite $2 \cdot 10^{-4}$ rad) beeinflußt. Berücksichtigt man dies durch Faltung, so stellt man eine gute UUbereinstimmung zwischen dem berechneten und dem beobachteten Verlauf fest.

1 C. Kunz u. H. Raether, Naturwissenschaften 48, 711 [1961]. - C. Kunz, Z. Phys. 167, 53 [1962].

2 E. A. Stern u. R. A. Ferrell, Phys. Rev. 120, 130 [1960]. 
Während die oben angegebene Verteilung für senkrechte Inzidenz gilt, sagt die Theorie der Oberflächenplasmaanregung ${ }^{2}$ für nichtsenkrechte Inzidenz eine für Oberflächenplasmaverluste charakteristische Unsymmetrie in der Winkelverteilung voraus: die Intensität der Oberflächenplasmaverluste soll zu beiden Seiten des Primärstrahles verschieden groß sein (vgl. Abb. 1). Diese Unsymmetrie ist wegen des raschen Abfalls der Intensität mit dem Winkel nur in sehr kleinen Winkeln beobachtbar und ist deshalb bisher noch nicht gefunden worden.

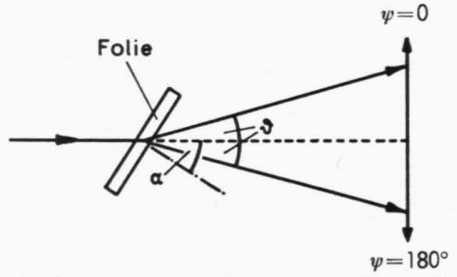

Abb. 1. Zur Definition der Winkel: $\alpha=$ Einfallswinkel, $\vartheta=$ Streuwinkel, $\psi=$ Azimut in der Beobachtungsebene.

Experimente am $5 \mathrm{eV}$-Verlust von Silicium und Germanium zeigten diese Unsymmetrie. Der Einfallswinkel betrug $\alpha=45^{\circ}$, und es wurden in der Einfallsebene in Winkeln bis $\vartheta=2,5 \cdot 10^{-4}$ zu beiden Seiten des Primärstrahls Energiespektren aufgenommen. Das Ergebnis zeigen die Meßpunkte in Abb. 2, in der die beobachteten Intensitäten des $5 \mathrm{eV}$-Verlustes dargestellt sind. Die elastische Primär-Intensität wurde für $\vartheta=0$ $1000 \%$ gesetzt.

Man kann einen Vergleich mit der Theorie durchführen, wenn man das endliche Winkelauflösungsvermögen (Halbwertsbreite ca. $1 \cdot 10^{-4} \mathrm{rad}$ ) berücksichtigt. Dies erreicht man durch numerische Faltungsintegration der theoretischen Winkelverteilung mit der Winkelverteilung des Primärstrahles. Das Ergebnis ist in Abb 2 als Kurve eingezeichnet, die an die Meßpunkte angepaßt ist. Man sieht - im Rahmen der Meßgenauigkeit - eine recht gute Übereinstimmung.

Die Voraussagen von Stern und Ferrell über die Eigenschaften der Oberflächenplasmaverluste sind hierdurch in zwei wesentlichen Punkten für den $5 \mathrm{eV}$-Verlust in Silicium und Germanium bestätigt worden. Eine ausführliche Beschreibung der Experimente erfolgt an anderer Stèlle.

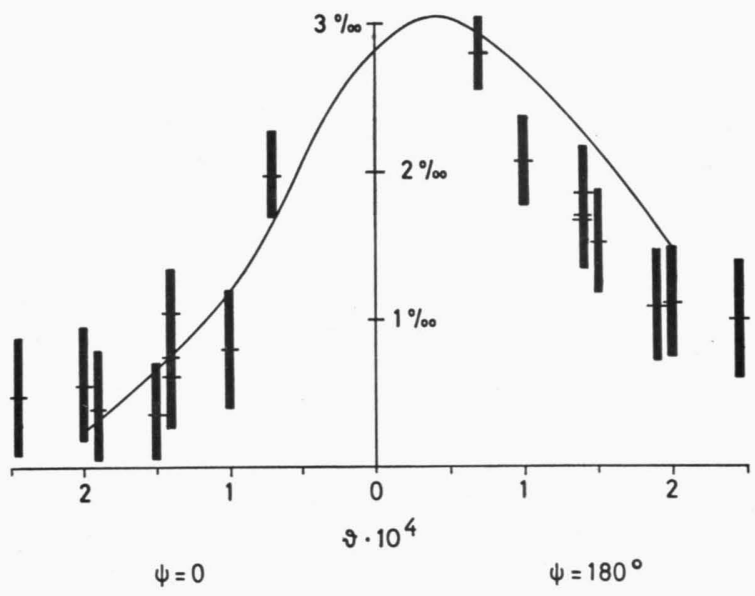

Abb. 2. Winkelverteilung der Intensität des $5 \mathrm{eV}$-Verlustes an Silicium und Germanium bei nichtsenkrechter Inzidenz. Man erkennt die Unsymmetrie bezüglich des Maximums des Primärstrahles $(\vartheta=0)$. Der große Fehler der Meßpunkte ist durch die Unsicherheit in der Abtrennung der Flächen unter dem 5 eV-Maximum vom Untergrund gegeben. Die ausgezogene Kurve stellt den theoretischen Verlauf dar.

Herrn Prof. Dr. H. RaEther danke ich herzlich für seine ständige Förderung. 